\title{
Application of a Smart Parachute Release Algorithm to the CPAS Test Architecture
}

\author{
Kristin J. Bledsoe ${ }^{1}$ \\ Jacobs Engineering, Houston, TX, 77058
}

\begin{abstract}
One of the primary test vehicles for the Capsule Parachute Assembly System (CPAS) is the Parachute Test Vehicle (PTV), a capsule-shaped structure similar to the Orion design but truncated to fit in the cargo area of a C-17 aircraft. The PTV has a full Orion-like parachute compartment and similar aerodynamics; however, because of the single-point attachment of the CPAS parachutes and the lack of an Orion-like Reaction Control System (RCS), the PTV has the potential to reach significant body rates. High body rates at the time of Drogue release may cause the PTV to flip while the Pilot parachutes deploy, which may result in the severing of Pilot or Main risers. In order to prevent high rates at the time of Drogue release, a "smart release" algorithm was implemented in the PTV avionics system. This algorithm, which was developed for the Orion flight system, triggers the Drogue parachute release when the body rates are near a minimum. This paper discusses the development and testing of the smart release algorithm; its implementation in the PTV avionics and the pre-test simulation; and the results of its use on two CPAS tests.
\end{abstract}

$\begin{array}{ll}\text { CPAS } & =\text { Capsule Parachute Assembly System } \\ \text { CPSS } & =\text { Cradle Platform Separation System } \\ \text { EDL } & =\text { Entry, Descent, and Landing } \\ \text { EDU } & =\text { Engineering Development Unit } \\ \text { LVAD } & =\text { Low Velocity Aerial Delivery } \\ \text { OF } & =\text { Oscillation Fraction } \\ \text { MSL } & =\text { Mean Sea Level } \\ \text { PROF } & =\text { Pitch Rate Oscillation Fraction } \\ \text { PTV } & =\text { Parachute Test Vehicle } \\ \text { RSS } & =\text { Root Sum of Squares } \\ \text { SDR } & =\text { Smart Drogue Release }\end{array}$

\section{Introduction}

$\mathrm{T}$ he Capsule Parachute Assembly System (CPAS) consists of 11 parachutes that are deployed during the Entry, Descent, and Landing (EDL) phase of the Orion capsule's mission to slow the capsule for safe splashdown. CPAS is being rigorously tested to demonstrate the successful deployment of all parachutes and to quantify the parachute performance in flight-like conditions. The primary method of testing is through Low-Velocity Aerial Delivery (LVAD) drop tests. ${ }^{1}$ During the current Engineering Development Unit (EDU) phase of testing, speciallydesigned test vehicles are extracted from an aircraft at 25,000 ft-Mean Sea Level (MSL), and a programmer parachute is used to achieve the desired conditions at Drogue parachute deployment. One of the primary test vehicles is the Parachute Test Vehicle (PTV). The PTV is a capsule shape similar to Orion, but truncated in height to fit within the cargo space of a C17 aircraft (Fig. 1) ${ }^{1}$. The PTV has a parachute compartment representative of that on Orion,

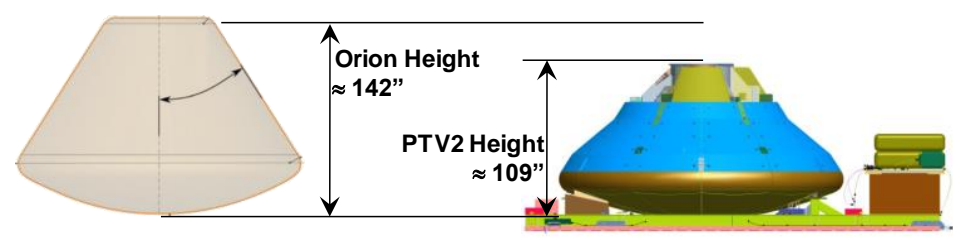

Figure 1. Comparison of Orion and PTV.

\footnotetext{
${ }^{1}$ Analysis Engineer, Aeroscience and Flight Dynamics, 2224 Bay Area Blvd, Houston, TX, AIAA Member
} 
allowing for the entire CPAS to be deployed during a test. This test vehicle is used to examine the performance of the CPAS parachutes in a capsule wake.

The PTV is aerodynamically unstable due to its capsule shape. It is less stable than the Orion capsule is predicted to be, due to the truncated height, and it lacks the reaction control thrusters of the Orion that can counteract aerodynamic forces. As a result, during a drop test, the PTV has a higher potential for building high body rates and achieving undesirable orientations than Orion. In certain extreme orientations, there is the risk that textile parachute risers will come into contact with the PTV forebody. While the PTV forebody is covered in foam, that foam can easily be penetrated by tensioned risers, exposing the sharp aluminum beneath. Contact with a sharp edge can sever textile risers, causing a loss of parachute and potentially resulting in test failure and loss of vehicle. This risk is highest after the release of the Drogue parachutes, when high rates can cause the PTV to flip over, significantly increasing the chance of Pilot or Main riser contact.

In order to reduce the likelihood of flipping after Drogue release, a Smart Drogue Release (SDR) algorithm was developed. SDR ensures that the Drogues are released when the PTV is at or near a minimum body rate. Because of the aerodynamic instability of the PTV shape at certain angles of attack, an additional criterion was later added to the SDR algorithm to ensure that the Drogues are released at or near a maximum angle of attack. The SDR logic was tested and implemented on the PTV avionics software and has been used successfully on two CPAS drop tests to date.

\section{Description of Algorithm}

The CPAS SDR algorithm is based on the algorithm developed by Lockheed Martin for Orion. At a high level, the Orion SDR algorithm operates as follows:

1) Calculate the root sum of squares (RSS) of the pitch and yaw rates.

2) If the rate is below a specified minimum, signal parachute release.

3) Detect peaks and troughs in the RSS data.

4) Compute the oscillation fraction.

5) If the current OF is lower than the specified trigger value, signal parachute release.

Minor modifications to this algorithm were made to account for the CPAS test vehicle avionics system, which differs from the Orion system. The PTV avionics system uses time, rather than altitude, as the basis for determining when to activate the SDR algorithm and contains a different suite of sensors. In addition, a second criterion based on pitch rate was added to ensure release at a maximum angle of attack.

\section{A. Operation}

In the original implementation, the parachute release command was to be issued at a minimum body rate alone. The SDR algorithm execution is intended to begin at PTV/Cradle Platform Separation System (CPSS) separation, and continually inspect the RSS of the NAV440 sensor's filtered pitch rate and yaw rate components. The algorithm detects the "peaks" and "troughs" in the data as shown in Figure 2. On each execution cycle beginning at the opening of the SDR time window, an "oscillation fraction" (OF) - the ratio of the current and trough rate difference to peak and trough rate difference - is computed as shown in Eq. (1):

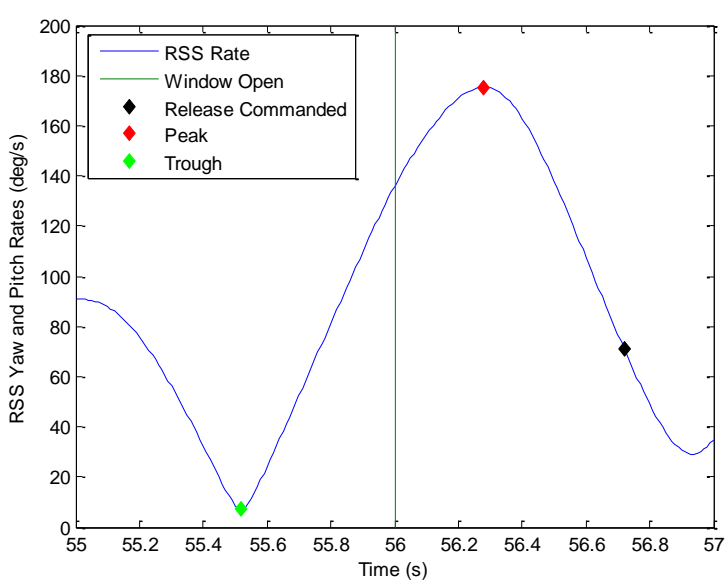

Figure 2. Example of peaks and troughs.

$$
O F=\frac{\text { current rate }- \text { trough rate }}{\text { peakrate }- \text { trough rate }}
$$

The release criterion is: a) the rate is lower than a specified minimum rate; $b$ ) the rate is decreasing and the $\mathrm{OF}$ is less than the descending trigger (defined as a target $\mathrm{OF}$ value below which the RSS rate criteria is met when the RSS rate is decreasing); or c) the rate is increasing and the $\mathrm{OF}$ is less than the ascending trigger (defined as a target $\mathrm{OF}$ value below which the RSS rate criteria is met when the RSS rate is increasing). In both cases $\mathrm{b}$ and $\mathrm{c}$, the OF must be small, meaning that the current rate must be close to the last recorded trough value.

Note that since an approaching minimum cannot be detected in real time, the previous trough must be used in the $\mathrm{OF}$ calculation. Therefore, if the system is underdamped on a given cycle, so that the approaching minimum would be

2

American Institute of Aeronautics and Astronautics 
lower than the last recorded trough, the computed OF trigger value is compromised and the release command would occur further from the upcoming trough than desired. Conversely, if the system is overdamped on a given cycle, the release command will occur closer to the upcoming trough; if there is sufficient damping, there is a chance that the trigger value will not be met before the trough is reached.

The original CPAS implementation of the SDR algorithm did not allow for algorithm start from the system initiation time; peaks and troughs were not recorded until the start of the SDR time window. This caused a delay of at least one cycle before the release would be commanded. It was decided to procede without changes for CDT-3-5 as the SDR time window was sufficiently large to accommodate the resulting expected delay.

Prior to CDT-3-7, a second criterion was added to also constrain the release on maximum angle of attack. The algorithm tracks the signed pitch rate for peaks and troughs, and a Pitch Rate Oscillation Fraction (PROF) is calculated as in Eq. (1). The criterion is met if both the rate is decreasing and the PROF is between the maximum and minimum PROF bounds.

Ideally, both criteria should be met concurrently to
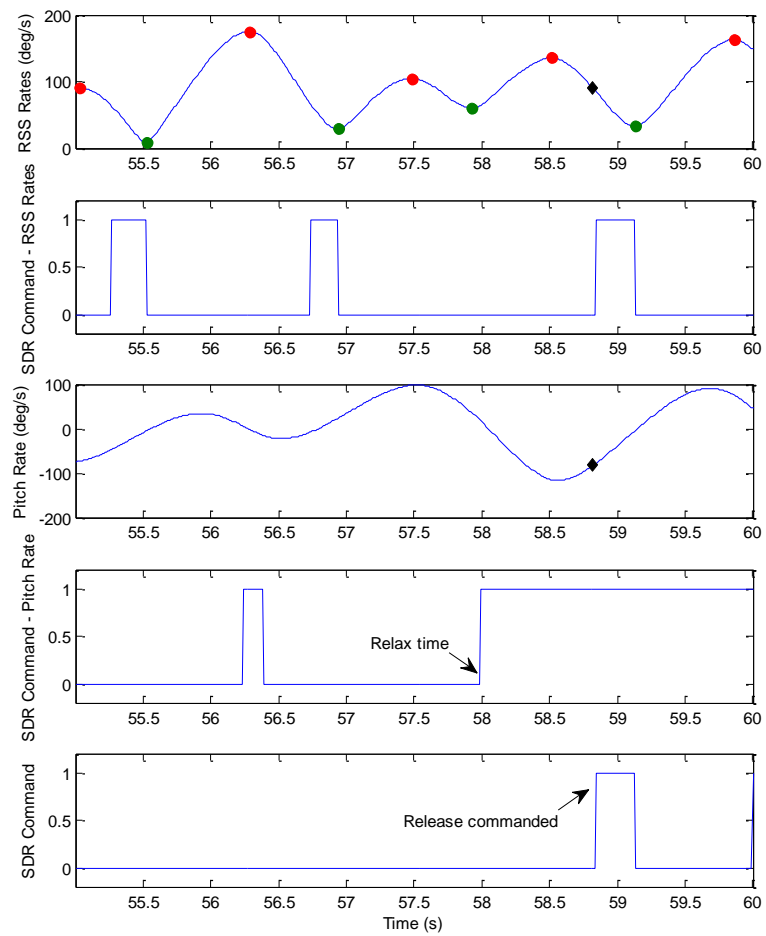

Figure 3. Example of relaxed pitch rate condition.

command parachute release. However, if the pitch and yaw rates are out of phase, it is possible that the criteria will not be met concurrently during the SDR time window. Therefore, after a specified time, the pitch rate criterion is "relaxed" or nulled, and release will be commanded on the RSS rate criterion alone (Fig. 3). If release conditions are not met within the SDR time window, release will be commanded at the end of the window, regardless of the rate at that time.

\section{B. Algorithm Inputs}

Window open: The time in seconds from system initiation (PTV/CPSS separation in the case of CPAS testing) when the SDR OF calculations begin and are compared to the triggers to determine if release should be commanded. Release will not be commanded prior to this time. Release may occur at the window open time if the release

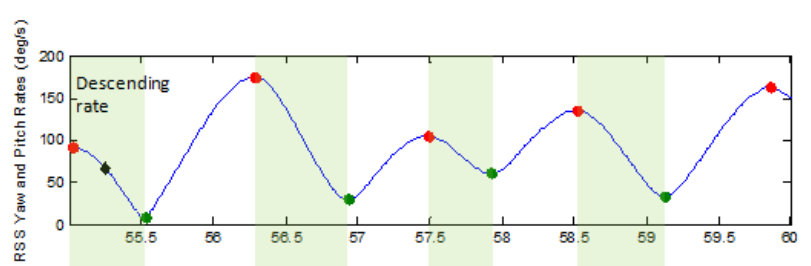
conditions have been met.

Window close: The time in seconds from system initiation when the SDR time window ends. If release has not been commanded by this time, it is commanded immediately.

Relax time: The time in seconds from system initiation when the pitch rate criterion is set aside or

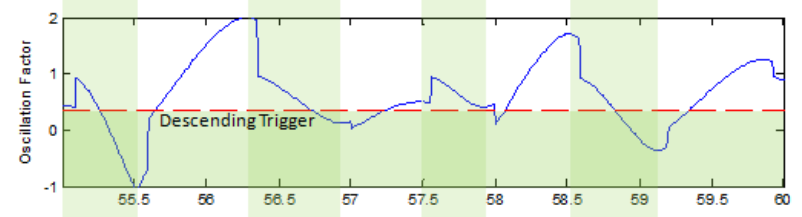
"relaxed." For the remainder of the SDR time window, only the RSS rate criterion must be met for release to be commanded. See Fig. 3.

Ascending trigger: The OF value below which the RSS rate criterion is met when the RSS rate is increasing. Can be set to a large negative number to

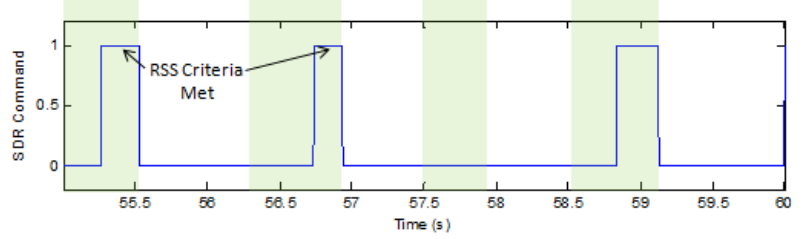

Figure 4. Descending trigger example. ensure that release will not be commanded when the RSS rate is increasing.

Descending trigger: The OF value below which the RSS rate criterion is met when the RSS rate is decreasing. Can be set to a large negative number to ensure that release will not be commanded when the RSS rate is decreasing. See Fig. 4. 
Pitch rate oscillation fraction bounds: The maximum and minimum PROF values at which the pitch rate criterion is met. See Fig. 5.

Minimum rate: The RSS rate below which release will be commanded regardless of whether the other criteria are met.

Threshold: If the denominator of the OF equation is too small (i.e. the peak and trough values are very close) and falls below the threshold, the OF will be set equal to 1 . This prevents the $\mathrm{OF}$ from becoming extremely large and potentially causing unexpected problems in the software.

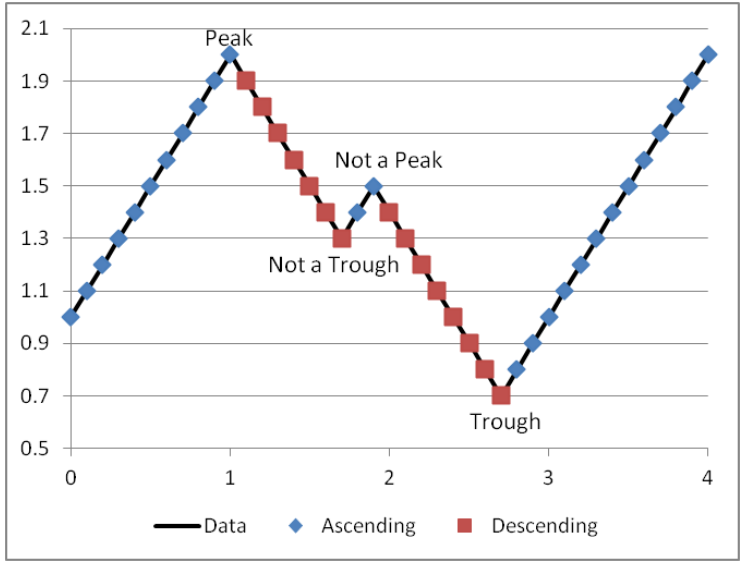

Figure 6. Example of persistence.
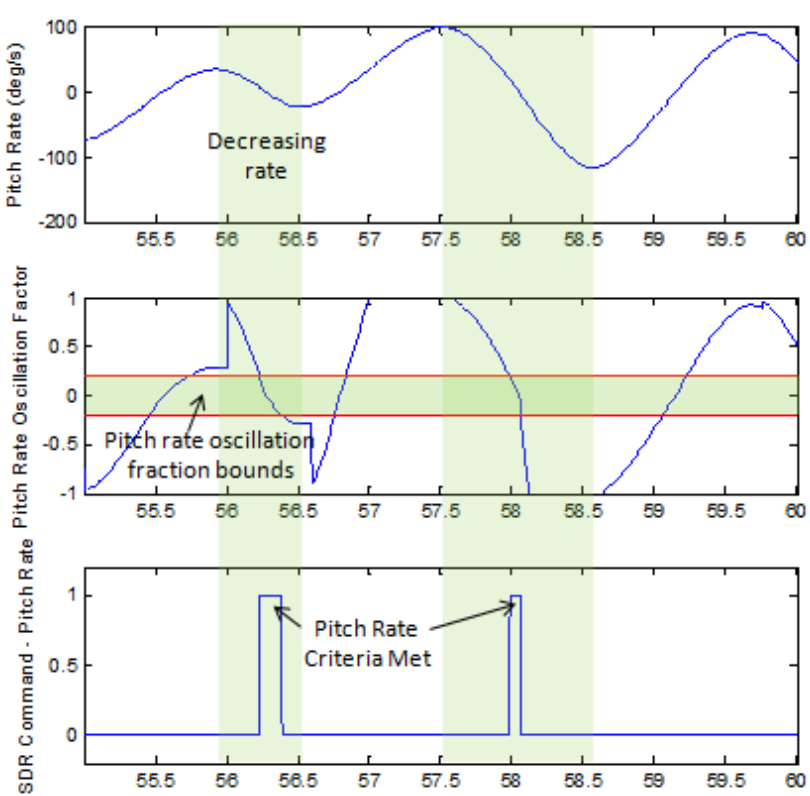

Figure 5. Pitch rate oscillation fraction example.

Persistence: The number of data points required to establish a peak or trough. If the persistence is a number P, there must be $\mathrm{P}$ data points with increasing rates followed by $\mathrm{P}$ data points with decreasing rates for a peak to be recorded. The example in Fig. 6 has a persistence greater than 3 but less than 8 .

\section{Algorithm Implementation}

The Orion SDR algorithm was delivered to CPAS as pseudocode. CPAS engineers used the psuedocode to develope two independent scripts, one in MATLAB and one in LabView, to test the algorithm and modify it for CPAS use. The two scripts were created independently to allow for rigorous testing of the algorithm, as each provided a check on the results of the other. Comparisons of the two sets of output were made until acceptable matches were achieved. The LabView script was then implemented on flight-like avionics for bench testing, and finally on the actual PTV avionics for final checkout and use on the drop test.

In each phase of implementation, the scripts were tested using data from past tests, data from simulations, and hand-generated data sets that covered additional "edge" cases that were not represented in the other data sets. In all cases, the MATLAB script was used to verify the results of the avionics software. The resulting trigger times for each data case were compared between the avionics and the MATLAB script. Differences of less than a tenth of a second were explained by system lag in the avionics. Larger discrepancies were found to be caused by the filtering used in the avionics and the different methods of handling persistence between the two scripts. The differences were accepted, and the avionics software was validated for use on the drop tests.

\section{Drop Test Performance}

\section{A. CDT-3-5}

The second PTV test in the EDU test series, called CDT-3-5, was the first use of the SDR algorithm on a CPAS drop test. ${ }^{2}$ Significant dynamics had been observed under the Drogue parachutes on the previous PTV test, CDT-3-3. Although other modifications had been implemented for CDT-3-5 to reduce the dynamics, SDR was intended to reduce the risk to the Pilots and Mains in the event that the other mitigations did not achieve a stable vehicle. 
Table I. SDR Algorithm Inputs for CDT-3-5

\begin{tabular}{|l|l|}
\hline Window Open & $70 \mathrm{~s}$ \\
\hline Window Close & $75 \mathrm{~s}$ \\
\hline Persistence & 8 \\
\hline Threshold & 0.01 \\
\hline Minimum Rate & 0.0 \\
\hline Ascending Trigger & -10000 \\
\hline Descending Trigger & 0.35 \\
\hline
\end{tabular}

The SDR algorithm used for CDT-3-5 only checked for the RSS rate criteria; the pitch rate criteria was not added until the next PTV drop test, CDT-3-7. Additionally, as previously stated, the implementation for CDT-3-5 did not begin checking for peaks and troughs in the RSS rate prior to the window open time. Because of this, release could not be commanded until a full cycle had been completed within the time window. This was a known deficiency in the implementation that would delay release by roughly one second.

The inputs to the SDR algorithm for CDT-3-5 were based the values developed for the Orion algorithm, as listed in Table I. These input values limited release to occur strictly on a decreasing rate, not on increasing rates or based on a specific minimum value. The descending trigger of 0.35 was intended to account for the lag in the Orion avionics system; an assumption was made that the CPAS avionics system would show a similar lag.

The PTV uses redundant avionics systems to ensure that events are commanded successfully. On CDT-3-5, the

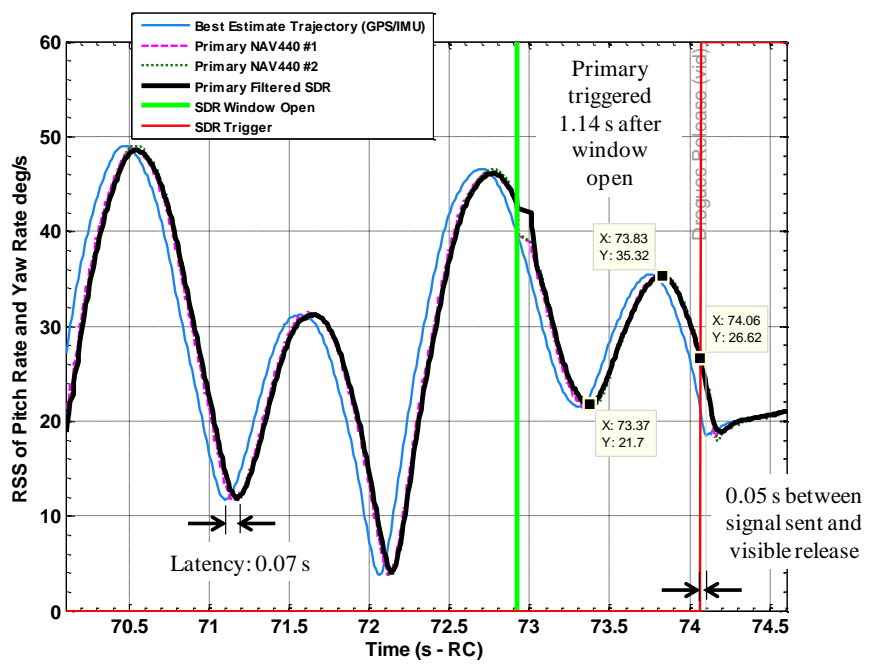

a)

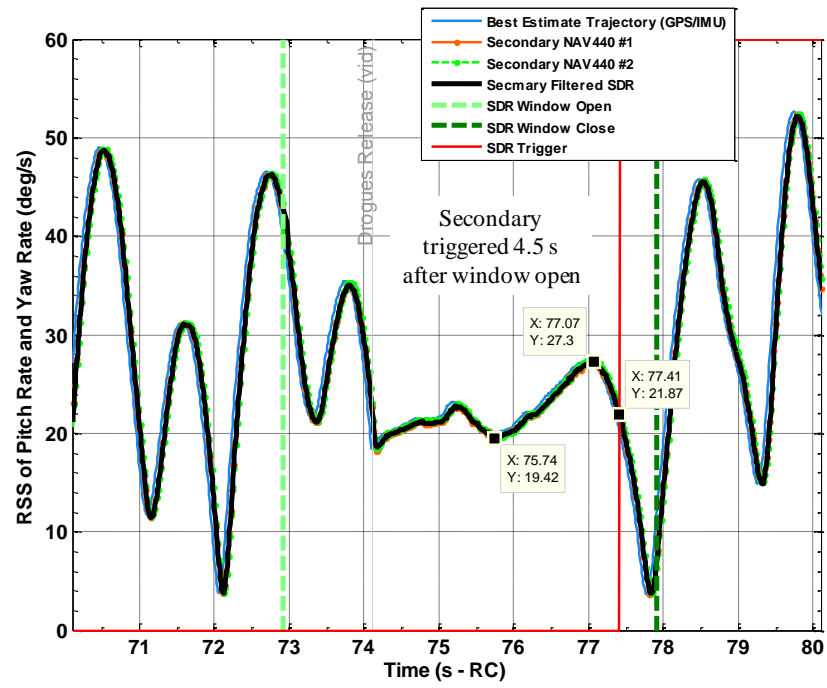

b)

Figure 7. SDR data for CDT-3-5: a) primary; b) secondary.

SDR functioned as expected on both the primary and secondary systems. The primary system commanded Drogue release $1.14 \mathrm{~s}$ after the window open (Fig. 7a). The calculated oscillation fraction at that time was 0.36 . There was a lag of $50 \mathrm{~ms}$ between the time that the command signal was sent and when Drogue release was visible; this is less than the expected lag of $70 \mathrm{~ms}$. The lag did not have an adverse effect on the release conditions, as the rates did not pass through a minimum and begin increasing prior to Drogue release.

The secondary system did not command release at the same time as the primary system. Due to noise in the data on the secondary system, the first peak was not detected. However, the secondary system did detect the next peak and trough. The secondary system commanded release $4.5 \mathrm{~s}$ after window open (Fig. $7 \mathrm{~b}$ ). The oscillation fraction at that time was 0.31 . This was a redundant signal, as release had already occurred. Note that the significant delay before the secondary system detected the subsequent peak and trough was caused by the sudden change in vehicle dynamics due to the successful release of the Drogue parachutes. The discrepancy between the primary and secondary systems exposed a new possible failure mode in the avionics due to a lack of independence between the two systems that is to be corrected in tests beyond CDT-3-7.

\section{B. CDT-3-7}

The third PTV test, called CDT-3-7, was intended to test a Drogue-out condition, wherein a single Drogue in the cluster of two failed to deploy. In this situation, the single remaining Drogue provides all of the drag and control authority on the vehicle until it is released and the Pilot and Main parachutes are deployed. The higher dynamic pressure and reduced control authority provided by a single Drogue made this test higher risk than CDT-3-5. 
Preflight trajectory simulations indicated a significant probability of high body rates and unfavorable orientations at Drogue release. The reduced control authority of the Drogue would allow the PTV to achieve lower angles of attack than previously seen. Aerodynamic assessments of the PTV showed that at angles of attack less than $100^{\circ}$, the PTV would flip towards apex-forward, rather than heatshield-forward, and the body rates would increase significantly. Monte Carlo assessments showed as much as a 36\% chance of parachute loss due to textile riser contact.

In order to reduce the potential for PTV flips and excessive body rates, the pitch rate criteria for SDR was added. By limiting Drogue release conditions to the maximum angle of attack on a given cycle, rather than on either the maximum or minimum, the risk of releasing at an aerodynamically unstable orientation was reduced. By implementing this additional criteria, the risk was more than halved. Additional modifications to the SDR inputs, refinements of the aerodynamics, and the addition of a second programmer parachute to improve the initial conditions of the Drogue phase, reduced the risk to less than $1 \%$.

CDT-3-7 was performed on December 20, 2012. A full data analysis has not been completed as of the writing of this paper. However, preliminary assessments indicate that SDR performed nominally.

\section{Conclusions}

Smart Drogue Release has been successfully implemented on the PTV for CPAS testing. The next step will be to implement smart release on the programmer parachutes as well, to ensure a clean handover at Drogue deploy. As testing becomes more complex, the SDR algorithm may be further refined or modified to ensure the safety of the test vehicle and the successful completion of the tests.

\section{Acknowledgments}

The author would like to acknowledge David Shoemaker, Charity Duke, and the other members of the Lockheed Martin Orion GNC team that developed the Orion SDR algorithm. Thanks also go to Kwaku Nornoo, Thomas Taylor, and the rest of the CPAS Avionics team for their work in implementing the SDR algorithm on the PTV avionics system.

\section{References}

${ }^{1}$ Ray, E., and Morris, A., "Challenges of CPAS Flight Testing,” AIAA 2011-2557, 2011.

${ }^{2}$ Ray, E., "CPAS EDU-A-CDT-3-5 Parachute Test Vehicle Analysis Report,” ESCG-8400-12-CPS-DOC-0104A, Jacobs Technology, Houston, TX, 2011. 